\title{
Observations of atmospheric gravity waves by radio interferometry: are results biased by the observational technique?
}

\author{
C. Mercier ${ }^{1}$, A. R. Jacobson ${ }^{2}$ \\ ${ }^{1}$ Observatoire de Paris-Meudon, URA 2080, France (e-mail: mercier@obspm.fr) \\ ${ }^{2}$ Los Alamos National Laboratory, New Mexico 87545, USA (e-mail: ajacobson@lanl.gov)
}

Received: 25 March 1996 / Revised: 4 December 1996 / Accepted: 13 December 1996

\begin{abstract}
In this paper we present a quantitative comparison between a large data base of medium-scale atmospheric gravity waves (AGWs) observed by radio interferometry of transionospheric radio sources and the results of a numerical simulation of the observed effects. The simulation includes: (i) the propagation and dissipation of AGWs up to ionospheric heights and (ii) the calculation of the subsequent slant TEC perturbations integrated along the path to the radio sources. We show that the observed azimuthal distribution of AGWs can be deeply biased. Predicted results are found to be consistent with previous extensive observations using radio beacons aboard geostationary satellites. These observations are rediscussed in view of the present predictions.
\end{abstract}

\section{Introduction}

Atmospheric gravity waves (AGWs) are a neutral-air phenomenon, but most techniques used to study them at ionospheric levels involve the effects they produce on the propagation of HF and VHF electromagnetic waves through perturbations in the electron density $n_{\mathrm{e}}$, because of the coupling between the motions of neutral air and ionisation through collisions. Among these techniques there is the particular case of transionospheric observations, giving effects integrated along the line of sight: measurements of Faraday rotation (e.g. Bertin et al., 1978) or of differential Doppler shifts of signals from satellite-borne beacons (Spoelstra and Kelder, 1984), interferometric observations of cosmic radio sources (Spoelstra and Kelder, 1984; Mercier, 1983, 1986, 1996; Jacobson et al., 1991; Jacobson and Erickson, 1992) and of satellite-borne beacons (Jacobson et al., 1995a, hereafter referred to as Jetal 95). Let us recall briefly what these techniques give access to.
- The Faraday rotation of a linearly polarised radio wave is proportional to the integral of the electron density along the line of sight, or slant TEC, and is scaled by $f^{-2}$, where $f$ is the used radio frequency. Using several receiving stations with mutual distances less than horizontal scale of AGWs, Bertin et al. (1978) obtained horizontal gradients of the slant TEC, parallel to the wave vector $\boldsymbol{K}$ of $\mathrm{AGWs}$ and hence their direction of propagation.

- The differential Doppler shifts between signals emitted at two frequencies from satellite-borne beacons is also proportional to the slant TEC along the line of sight. The motion of the satellite can also be used to derive the TEC gradient in the direction of this motion (Spoelstra and Kelder, 1984). Van Velthoven et al. (1990), using NNSS satellites with polar orbits, derived additionally the EW gradient of the slant TEC from three receiving stations shifted in longitude.

- The nature of the effects produced on ground-based interferometric observations of radio sources depends on the radio-frequency. At frequencies $f<20-50 \mathrm{MHz}$ the deviations of radio waves, scaled by $f^{-2}$, can be large enough to produce focusing effects. At frequencies $f>100 \mathrm{MHz}$ deviations of radio waves are generally small $(<$ some $\operatorname{arc} / \mathrm{min})$ and the amplitude of radio waves is unaffected, while the changes in their phases at antennas are proportional to the changes in slant TEC along paths towards the source. This last case is addressed in the papers already mentioned, and in the present one. If the ratio $r$ of the size of the array to the horizontal scale of AGWs is $\ll 1$ ("short" array), one gets only the horizontal gradient $\boldsymbol{G}$ of the slant TEC (Mercier, 1986), which gives propagation direction modulo $180^{\circ}$ for directional AGWs. The horizontal wavelength $\lambda$ cannot be derived with short arrays, except in special cases when additional observations giving information on extended parts of the wave are simultaneously available, e.g. focusing effects on the same radio source in the $20-50 \mathrm{MHz}$ range (Mercier et al., 1989) or differential Doppler shifts from rapidly moving satellites (Spoelstra, 1992). If $r \sim 1$ ("large" arrays) both 
the amplitude $A$ of AGWs and their horizontal wavelength $\lambda$ (Jacobson and Erickson, 1992) can be derived, allowing a more complete description of AGWs.

In summary, these techniques basically give access to the slant TEC along the line of sight towards the radio source and to its horizontal derivatives (in practice the first and/or the second ones); they have been used for statistical studies on extensive data bases. However, it was shown by Bertel et al. (1976) that the line-of-sightintegrated response in TEC is highly anisotropic, i.e. the amplitude of the observed effects depends strongly on the relative geometry of the AGWs and of the line of sight. The reason is that contributions of all points of the line of sight are not necessarily in phase. Thus a bias is a priori introduced in the observed amplitudes. As already noted by Mercier (1986), if one deals with only one plane $A G W$ at a time, geometrical characteristics such as horizontal wavelength, azimuth of propagation and trace speed are not altered. But even in this case results on the statistical distribution of AGWs vs. these geometrical characteristics can be biased, since the necessary condition for identifying an AGW is that its amplitude be above the incoherent background; thus the observation system may be blind to parts of the AGW population. This is particularly likely in the case where only few lines of sight are used, i.e. in the case of geosynchronous satellite-borne beacons.

Bertel et al. (1976) developed a method to simulate TEC perturbations due to waves with known parameters. This method, involving the WKB approximation, takes into account the dissipation of AGWs due to thermoconduction and (although not formally) to viscosity at ionospheric heights. Bertel et al. (1976) used the results of their simulation to discuss their observations of Faraday rotation of signals from a geosynchronous satellite. Mercier (1996) developed a similar simulation to discuss his interferometric observations of several cosmic radio sources. He had a large sample of line-ofsight azimuths and elevations, but no access to the horizontal wavelength $\lambda$ of AGWs (which is a key parameter in the simulation) since he used a "short" array. Nevertheless, assuming values typical of mediumscale AGWs for $\lambda$, he concluded that its main statistical results were not grossly affected.

Jetal 95 used an array specially designed for interferometry on signals at $136 \mathrm{MHz}$ from beacons aboard several geosynchronous satellites (GOES-2, ATS-3, ATS-1, GOES-3). The size of the array (initially $\sim 80 \mathrm{~km}$, later extended to $\sim 120 \mathrm{~km}$ ) was large enough to allow them to infer values for $\lambda$. Additionally, they had by far the largest data base in this type of study. However, because of the use of geosynchronous satellites, the range of lines of sight was small and observational biases in their results could not be ruled out.

The aim of this study is to investigate the possible resulting biases in statistical results from radio-interferometric observations, using the simulation code developed by Mercier (1996), and to rediscuss the results of Jetal 95, using the same data base extended to May 1996. In Sect. 2 we present the simulation model and in Sect. 3 the data base. In Sect. 4 we describe the method used for comparing the azimuthal distribution of observed AGWs and the predictions of the model. In Sect. 5 we give preliminary statistical results of observations, useful for the method described in Sect. 4. In Sect. 6 we compare observational results with predictions of the simulation. Finally in Sect. 7 we present the discussion and conclusions.

\subsection{Notation}

For clarity we define here notation used in the study:

$\alpha_{\text {wave }}$ azimuth of AGWs (deg. E of $\mathrm{N}$ ),

$\alpha_{l o} \quad$ azimuth of the line of sight towards satellites $(\operatorname{deg} \mathrm{E}$ of $\mathrm{N})$,

$A_{\text {obs }} \quad$ observed amplitude of TEC perturbations $\left(10^{14} \mathrm{~m}^{-2}\right)$,

$a_{\mathrm{fs}} \quad$ average value of the angle between the wave front and the line of sight ( $>0$ is the 1.o.s. from below to above the wave front),

$e_{\mathrm{ls}} \quad$ elevation of the line of sight (deg),

$f \quad$ frequency of AGWs $(\mathrm{mHz})$,

$f_{\text {coher }}$ coherence factor of integration along the line of sight $(\%)$,

$k_{\mathrm{h}} \quad$ horizontal wavenumber $\left(\mathrm{m}^{-1}\right)$,

$\lambda$ horizontal wavelength of AGWs $(\mathrm{km})$,

$V_{\text {n0 }} \quad$ horizontal neutral-wind velocity $\left(\mathrm{m} \mathrm{s}^{-1}\right)$,

$V_{\phi} \quad$ horizontal trace speed of AGWs $\left(\mathrm{m} \mathrm{s}^{-1}\right)$,

$z_{\text {launch }}$ launch altitude of AGWs (usually $20 \mathrm{~km}$ ),

$L \quad$ azimuthal amplitude response in TEC perturbation.

\section{The simulation model}

The method for simulating TEC perturbations is presented in Mercier (1996) and is similar to that developed by Bertel et al. (1976). It uses the theory of Volland (1969a) which includes the effects of thermal conduction (but not of ion drag) on AGW dissipation, and assumes WKB approximation to be valid for describing propagation in a non-isothermal and stratified medium (Volland, 1969b). Given a model of the unperturbed neutral atmosphere (Alcaydé, 1981) completed by a model of thermal conduction for atomic oxygen (Dalgarno and Smith, 1962), the main constituent above $150 \mathrm{~km}$, and a model of undisturbed neutralwind velocity $V_{\mathrm{n} 0}$ (Hedin et al., 1988), the complex vertical wave number $k_{z c}=k_{z r}+i k_{z i}$ can be derived for any AGW with given frequency $f$ and horizontal wavenumber $k_{\mathrm{h}}=2 \pi / \lambda$. Given the amplitude of waves at an initial altitude $z_{\text {launch }}$ this allows us to calculate their amplitude at any higher level (as long as they do not become evanescent). The perturbation $n_{\mathrm{e}}$ in the undisturbed electron density $n_{\mathrm{e} 0}$ is obtained by solving the continuity equation for $n_{\mathrm{e}}$, assuming that the perturbed ionisation velocity $V_{\mathrm{i} 1}$ is merely the projection of the perturbed neutral velocity $V_{\mathrm{n} 1}$ on the magnetic field lines. The perturbation $\mathrm{TEC}_{1}$ in the unperturbed slant $\mathrm{TEC}_{0}$ follows by integration along the line of sight. 
Waves are launched at a given altitude $z_{\text {launch }}$ and their propagation is calculated up to ionospheric levels. We do not intend to describe the generation mechanism of waves, but only to calculate the perturbations in electron density due to waves with given parameters. Thus only the amplitude of waves at ionospheric levels is of interest. The detail of their story at lower altitudes, say below the basis of the $\mathrm{F}$ region, can be ignored and we could take their amplitude at this level as a boundary condition, irrespective of their actual height of generation, all the more since the origin of medium-scale AGWs observed at mid-latitudes is poorly known. However, we took generally $z_{\text {launch }}=20 \mathrm{~km}$ (the lower limit of our model of undisturbed atmosphere), partly for convenience but also because of a possible origin at low altitude for waves. In some cases it turned out that waves with the observed $f$ and $l$ were evanescent. This can namely happen in the two following cases:

1) the frequency $f$ of the wave exceeds the Brunt-Väisälä frequency $f_{\mathrm{g}}=w_{\mathrm{g}} / 2 \pi$ at some level;

2) $f<f_{\mathrm{g}}$ but the local sound speed $c_{\mathrm{s}}$ is not large enough compared to the horizontal trace speed $V_{\phi}$ of the wave, before reaching the $\mathrm{F}$ region. This occurs preferentially at $z \sim 80 \mathrm{~km}$, where the temperature is minimum. It can then be argued that the actual $z_{\text {launch }}$ for these waves must be above this evanescence level. Such cases were presently discarded from our statistics.

The initial amplitudes of waves in the simulation were taken to be identical, and scaled by an overall multiplicative constant in such way that perturbations in slant TEC were on the average of the same order as the observed ones. The corresponding calculated values for $V_{\mathrm{nl}}$ are in the range $10-20 \mathrm{~m} \mathrm{~s}^{-1}$ at $z \sim 250 \mathrm{~km}$. It was checked that in no case do the waves break down, i.e. the perturbed local temperature gradient is nowhere lower than the adiabatic one.

We also derive two parameters of interest when discussing the slant TEC perturbations due to AGWs:

- The angle $a_{\mathrm{fs}}$ between the wave front and the line of sight. Since parameters of the unperturbed atmosphere are height dependent, wave fronts are not planar but curve up and $a_{\mathrm{fs}}$ changes typically by $\sim 35^{\circ}$ between 100 and $400 \mathrm{~km}$. The values used for $a_{\mathrm{fs}}$ in the following are averaged ones, taking $\left|d n_{\mathrm{e}}\right|$ as the weighting function of altitude $z$.

- The coherence factor $f_{\text {coher }}$ giving the efficiency of integration of $d n_{\mathrm{e}}$ along the line of sight. It is defined as:

$f_{\text {coher }}=\frac{\left|\int n_{\mathrm{el}} d l\right|}{\int\left|n_{\mathrm{el}}\right| d l}$,

where the integration path is along the line of sight. This coherence factor is a very sensitive function of the relative geometry of the wave and of the line of sight [see examples in Mercier (1996)]. It is thus meaningless to reduce the perturbation in the slant TEC (which depends in a complicated way on the line-of-sight orientation) to a perturbation in the vertical TEC, and throughout this paper we will always speak of perturbations in the slant TEC.
Waves are locally described in the approximation of flat earth, i.e. they are assumed to follow the curvature of the ground, and the integration is carried out taking into account the actual geometry of the straight line of sight and of the curved ground. Thus TEC perturbations for lines of sight with very low elevations, for which the vertical direction changes substantially across the ionosphere, can be properly obtained.

Let us briefly recall qualitatively the results of Mercier (1996): it was found that the amplitude of the response $L$ in slant TEC is very anisotropic and depends strongly on the difference between the pointing azimuth $\alpha_{\mathrm{lo}}$ and the propagation azimuth of the wave $\alpha_{\text {wave }}$. For moderate and high line-of-sight elevations, $L$ is restricted to a wide $\alpha_{\text {wave }}$ range in the same half plane as $\alpha_{10}$. For low and very low elevations $\left(<25^{\circ}\right)$ it becomes more anisotropic, with two large and narrow maxima for $\alpha_{\text {wave }}$ at $\sim 60^{\circ}$ on each side of $a_{\text {ls }}$ [see Fig. A2 in Mercier (1996)] and a central depletion for $\alpha_{\text {wave }} \sim \alpha_{\text {lo }}$. These two lateral maxima correspond roughly to the line of sight being parallel to the wave front. The neutral wind can complicate this description, its main effect being a stronger dissipation of waves propagating in the same direction.

\section{The data base}

The database is an extension of that already used by Jetal 95. It consists of more than 3 years (January 1993May 1996) of round-the-clock observations of four geosynchronous satellites (ATS-1, ATS-3, GOES-2, GOES-3) with the dedicated interferometer of Los Alamos. Total ranges and typical daily variations of azimuth and elevation of the lines of sights from Los Alamos to satellites are given in Table 1. The large total azimuthal ranges of GOES-3 and ATS- 1 are due to slow longitudinal drifts of these satellites. Except for ATS-1, all satellites are in or adjoin the SW quadrant of the sky. Elevations are essentially in the range $15^{\circ}-60^{\circ}$, with very low values for GOES-2 after 10 April 1995. As we will see later, these last observations will be particularly useful to constrain the predictions of the simulation. In the following we will refer to GOES-2 during the first period (up to December 1994) as GOES-2_93-94 and during the second period (after April 95) as GOES-2_9596.

Table 1. Azimuths and elevations of the lines of sight from Los Alamos to satellites used in this study

\begin{tabular}{lllll}
\hline satellite & $\begin{array}{l}\text { total ranges } \\
\text { azimuth } \\
\text { (deg E of N) }\end{array}$ & $\begin{array}{l}\text { typical daily variations } \\
\text { elevations } \\
\text { (deg) }\end{array}$ & $\begin{array}{l}\text { azimuth } \\
\text { (deg) }\end{array}$ & $\begin{array}{l}\text { elevation } \\
\text { (deg) }\end{array}$ \\
\hline GOES-2_93-94 & $215-235$ & $28-48$ & 18 & 20 \\
GOES-2_95-96 & $247-268$ & $01-17$ & 13 & 16 \\
ATS-3 & $176-180$ & $33-65$ & 4 & 35 \\
ATS-1 & $100-145$ & $15-60$ & 25 & 25 \\
GOES-3 & $170-250$ & $20-60$ & 12 & 15 \\
\hline
\end{tabular}


The selection of events has already been described by Jetal 95 . We only recall that propagating sinusoidal TEC perturbations are sought in the horizontal Fouriertransformed data. The selection criterion is that their amplitude is larger by at least $50 \%$ than that of the background. About 37000 events were thus selected. It must be noted they are not independent events, but rather often successive "snapshots" of a much smaller (maybe by an order of magnitude) number of wave trains. All events are gathered in a file with their main parameters: date, time elevation and azimuth of the satellite used, observed amplitude, wave azimuth, wavelength, frequency... In the following we will focus on typical medium-scale AGWs and will restrict to events with $0.4 \mathrm{mHz}<f<1.2 \mathrm{mHz}, 100 \mathrm{~km}<\lambda<400 \mathrm{~km}$ and $V_{\phi}<200 \mathrm{~m} \mathrm{~s}^{-1}$. The upper limit of $1.2 \mathrm{mHz}$ is taken slightly less than a typical value of Brunt-Väisälä frequency $f_{\mathrm{g}}$ in the $\mathrm{F}_{2}$ region $\left(f_{\mathrm{g}}=1.27 \mathrm{mHz}\right.$ for $T=1000 \mathrm{~K})$.

\section{Method}

Our first idea for investigating possible biases in the observed azimuthal distributions of AGWs was to feed the simulation code with a population of waves with the same frequencies $f$, horizontal wavelengths $\lambda$ and azimuths of propagation $\alpha_{\text {wave }}$ as the observed ones, but with uniform amplitudes, to check if there was a correlation between the predicted and observed amplitudes. A strong correlation was expected if the variations in the observed amplitudes were essentially due to the observational technique rather than to the natural scatter in the amplitudes of the waves. However this was
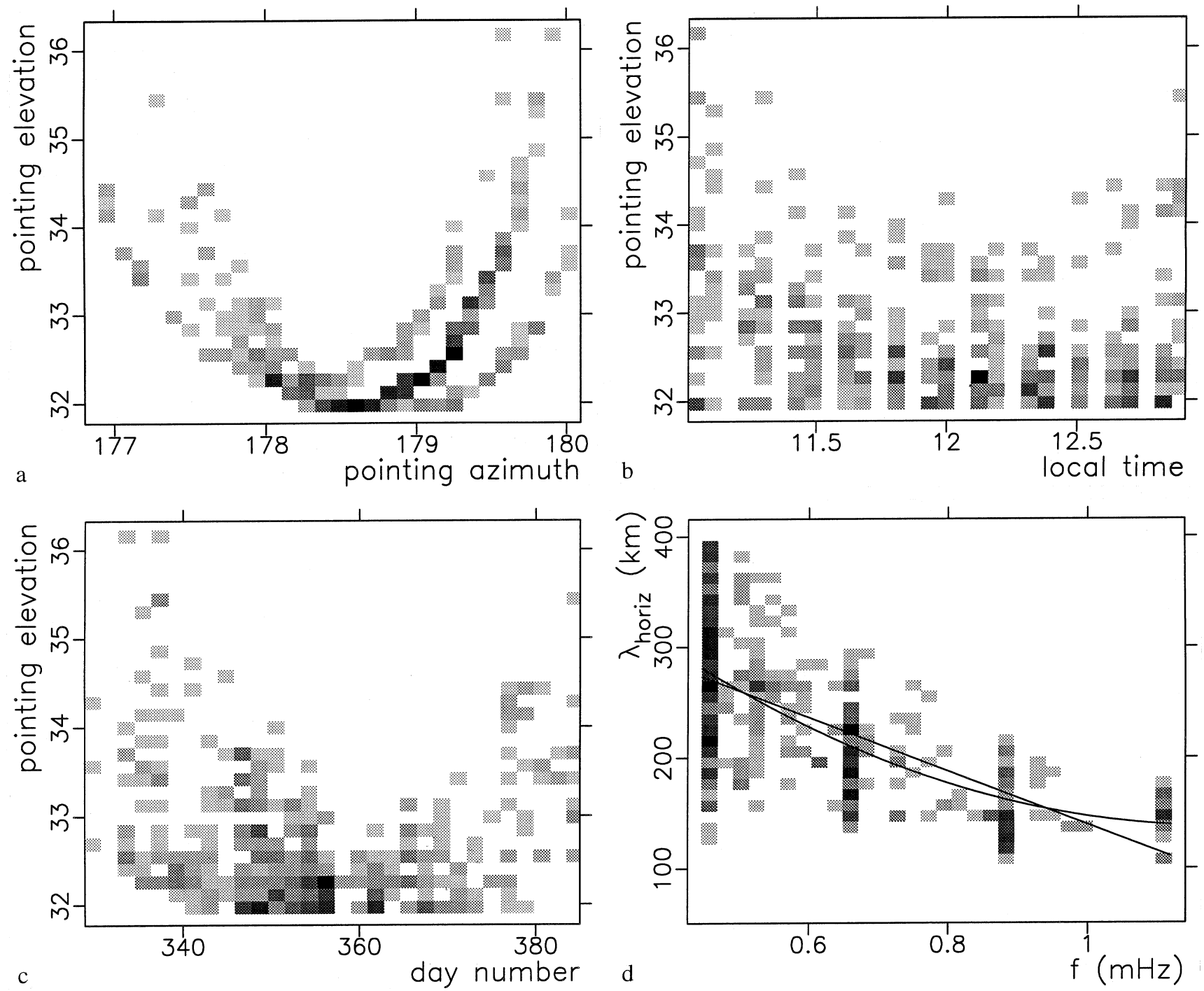

Fig. 1a-d. Observing conditions and results for 411 accepted (nonevanescent) events in the ranges $0.4<f<1.2 \mathrm{mHz}, 100<\lambda<$ $400 \mathrm{~km}$ and $V_{\phi}<200 \mathrm{~m} \mathrm{~s}^{-1}$, observed with ATS-3 in winter (Nov 25-Jan 20) for years 1993-1996, between 11 and 13 LT. There were 10 rejected (evanescent) events $(2 \%)$. Plots are grey-scale histograms of scatter plots; there are 9 intermediate grey levels determined by linear

interpolation between white (no events) and black. a gives azimuth vs. elevation of the line of sight, $\mathbf{b}$ and $\mathbf{c}$ the distribution of elevation of the line of sight with local time and day in the year. d displays wavelength $\lambda$ vs. frequency $f$; superimposed curves are fits to firstand second-order polynomials 
found not to be the case, the natural scatter in wave amplitudes destroying the possible correlation between the observed amplitudes and those predicted for a population with a uniform amplitude.

Hence we have adopted the following procedure:

- we consider subsets of data corresponding to chosen observing conditions (line of sight, local time and season) and wave parameters typical of medium-scale AGWs (defined in Sect. 3);

- we define the directional sensitivity (or "lobe") $L$, for the chosen observing conditions, as the relative variation in the predicted amplitude of the TEC perturbation with the azimuth $\alpha_{\text {wave }}$ of a causative wave, using values for $f$ and $\lambda$ typical of the observed AGWs ( $f=0.7 \mathrm{mHz}$ and $\lambda=200 \mathrm{~km}$; see Sect. 5);

- we then compare the lobe $L$ with the scatter plot of amplitude vs. azimuth for the AGWs in the selected subset.

It is then expected that the lobe must behave as an "envelope" for the observed distribution. More precisely, if the detection of AGWs is limited by the observational technique, the cut off in the observed distribution must coincide with that of the lobe; in this case we will speak of "truncation" of the distribution or of "complete filling" of the lobe. Conversely, if the observed population of AGWs is naturally restricted to some range of $\alpha_{\text {wave }}$, the observed distribution is expected to drop before the predicted lobe and we will speak of "incomplete filling" of the lobe.

The comparison must be made within samples of data sufficiently narrow for the observing conditions to be homogeneous since: (i) the predicted lobe depends both on wave parameters and (sensitively) on the set of observing conditions, and (ii) observed amplitudes exhibit a strong dependence on local time and season (e.g. Jetal 95; Mercier, 1996). Such small variations in observing conditions are all the more necessary since there can be systematic relationships between observing parameters, which can produce unexpected biases (for instance elevation of the line of sight is related to local time, with a slow shift during a year) and we must be very cautious in the interpretation of scatter plots spanning large variations of "hidden parameters".

The choice of the observing conditions in data subsets follows from the results of Jetal 95 on diurnal and seasonal behaviour of AGWs, which we recall here briefly. They showed the existence of two populations for AGWs: the first $\left(P_{1}\right)$ is observed from 8 to $16 \mathrm{LT}$, preferentially in winter, with propagation azimuths roughly towards $\mathrm{S}$; the second $\left(P_{2}\right)$ is observed between 13 and 22 LT, preferentially in summer, with propagation azimuths roughly towards W-NW. They found no evidence of diurnal rotation of propagation azimuths of AGWs within each of these two populations.

In the following we took intervals of $2 \mathrm{~h}$ around 12 and 18 LT and seasonal intervals of $\sim 2-3$ months in winter and summer as representative. The exact limits of these intervals were taken in each case as to minimise variations in pointing elevation and azimuth, as compromises between opposite requirements: the intervals must be short enough that observing conditions (position of the line of sight and climatology) do not change too much, and they must contain enough events for statistics to be significant.

\section{Preliminary statistical results}

The simulation needs the frequency $f$ and the horizontal wavelength $\lambda$ of the causative wave (the propagation azimuth $\alpha_{\text {wave }}$ is considered as a free parameter). In this section we present statistics on the observing conditions, and we derive from our observations a relationship

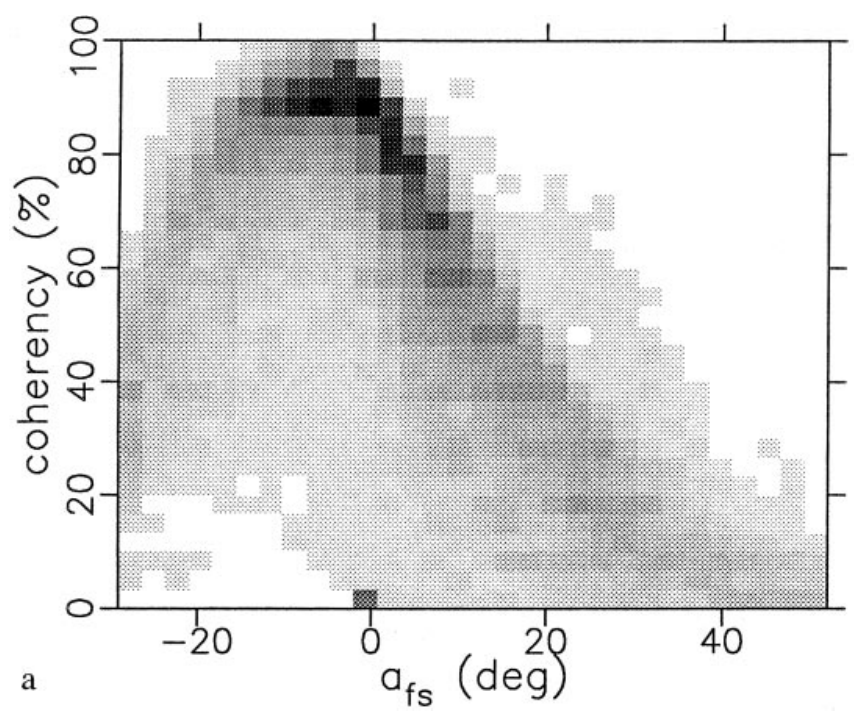

Fig. 2a, b. Grey-scale histograms of scatter plots for all events (all satellites) with parameters: $0.4<f<1.2 \mathrm{mHz}, 100<\lambda<400 \mathrm{~km}$ and $V_{\phi}<200 \mathrm{~m} \mathrm{~s}^{-1}$. There are 20672 accepted events and 1137 evanescent and rejected events $(5 \%)$ : a calculated coherency factor

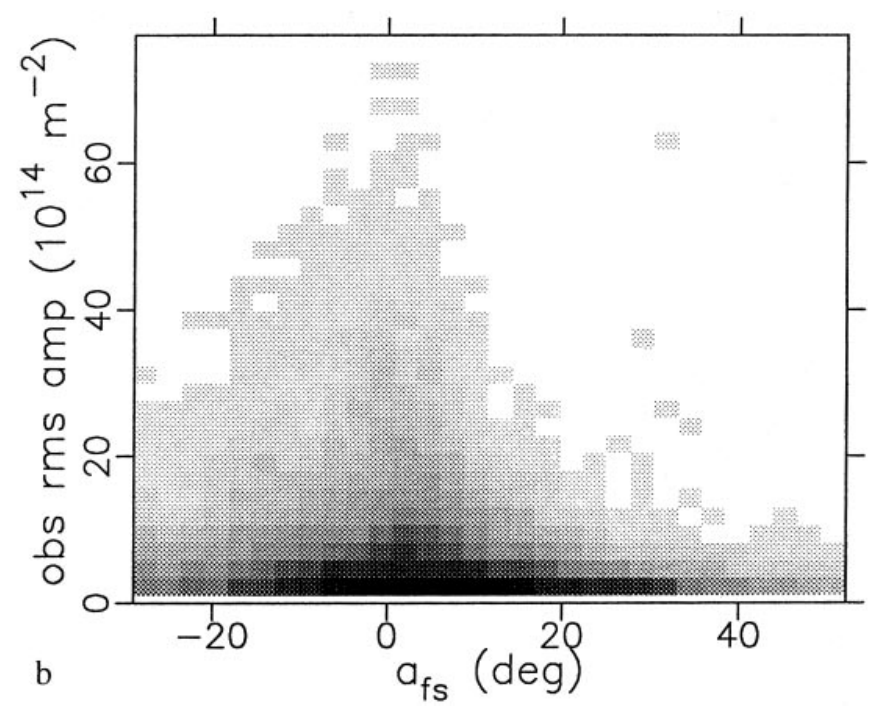

$f_{\text {coher }}$ versus calculated angle $a_{\mathrm{fs}}$ between the wave front and the one of sight ( $a_{\mathrm{fs}}>0$ when the line of sight is steeper than the wave front); b observed rms amplitudes vs. $a_{\mathrm{fs}}$ 


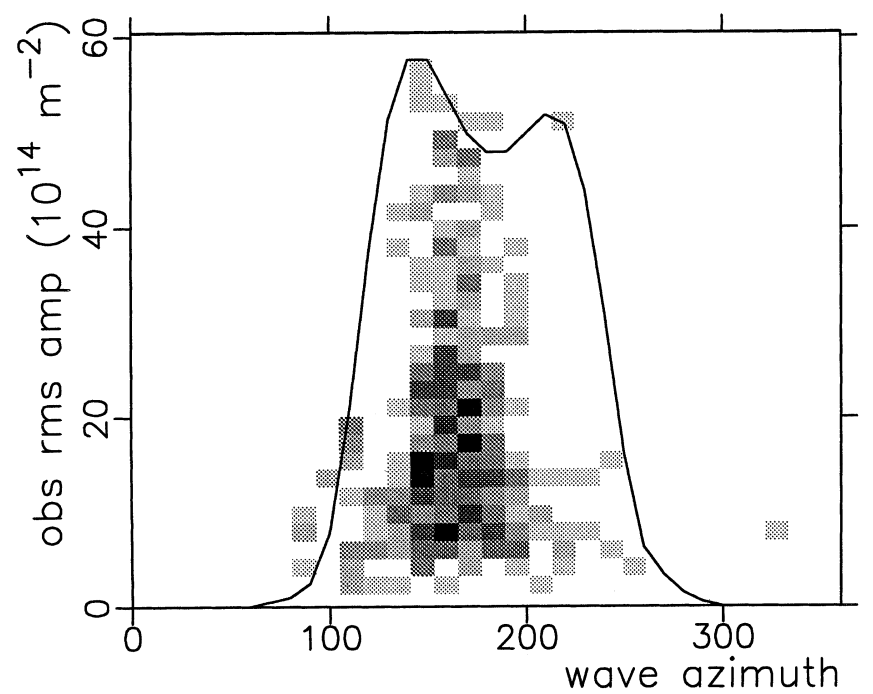

411 accepted events,
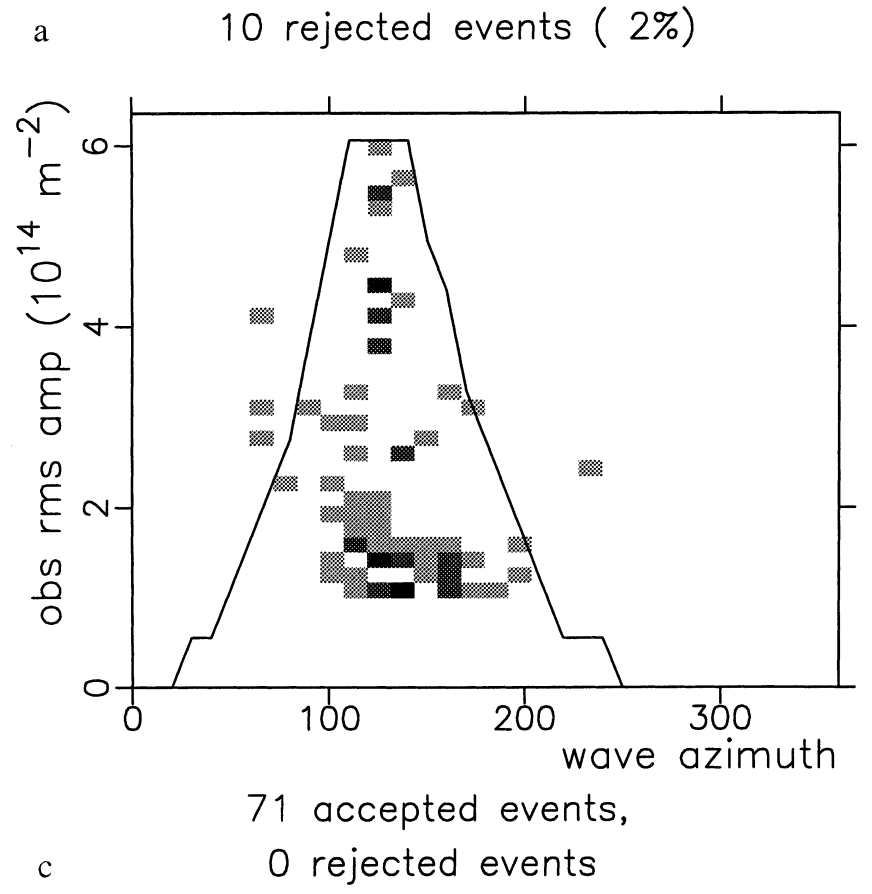

Fig. 3a-d. Grey scale histograms of the azimuthal distribution of events $(0.4 \mathrm{mHz}<f<1.2 \mathrm{mHz})$ observed with ATS-3: a winter 1113 LT; b winter 17-19 LT; c summer 11-13 LT; d summer 17-19 LT. Superimposed curves give the lobe (directional sensitivity of the

between $f$ and $\lambda$, which in principle is not biased by the observational technique.

Figure 1 gives observing conditions and observational results for the events observed by ATS-3 in the ranges $0.4<f<1.2 \mathrm{mHz}, 100<\lambda<400 \mathrm{~km}, V_{\phi}<200 \mathrm{~m} \mathrm{~s}^{-1}$, typical of medium-scale AGWs, in wintertime (25 November-20 January) between 11 and 13 LT. The line-of-sight orientation changes by only few degrees, but shows a systematic elevation change with the day in the year. The average wavelength $\lambda$ decreases with frequency $f$ from $270 \mathrm{~km}$ at $0.5 \mathrm{mHz}$ to $110 \mathrm{~km}$ at $1.2 \mathrm{mHz}$, with some dispersion. The same $(f, \lambda)$ relation is obtained between 17 and 19 LT, but from
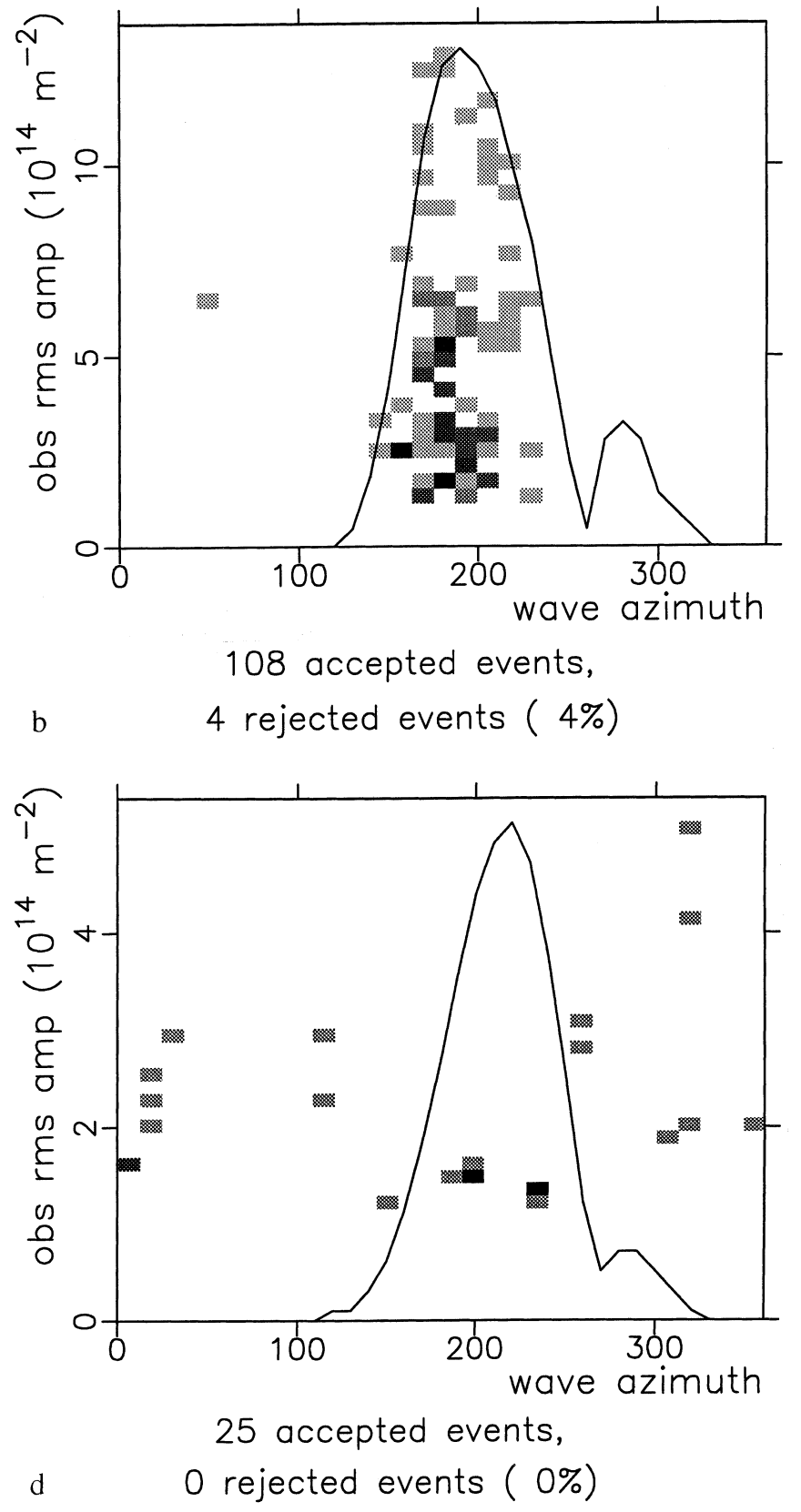

observation) for the average line of sight and a typical wave $(f=0.7 \mathrm{mHz}, \lambda=200 \mathrm{~km})$. The numbers of accepted and rejected events are indicated

fewer events. Similar relations are obtained from GOES2. In the following we take $f=0.7 \mathrm{mHz}$ and $\lambda=200 \mathrm{~km}$ as typical AGW parameters for computing lobes.

\section{Comparison of observation with simulation calculations}

\subsection{Relative position of wave fronts and lines of sights; efficiency of the integration}

For each observed wave we calculate the angle $a_{\mathrm{fs}}$ between the wave front and the line of sight, and also the coherence factor $f_{\text {coher }}$. These quantities are averaged 

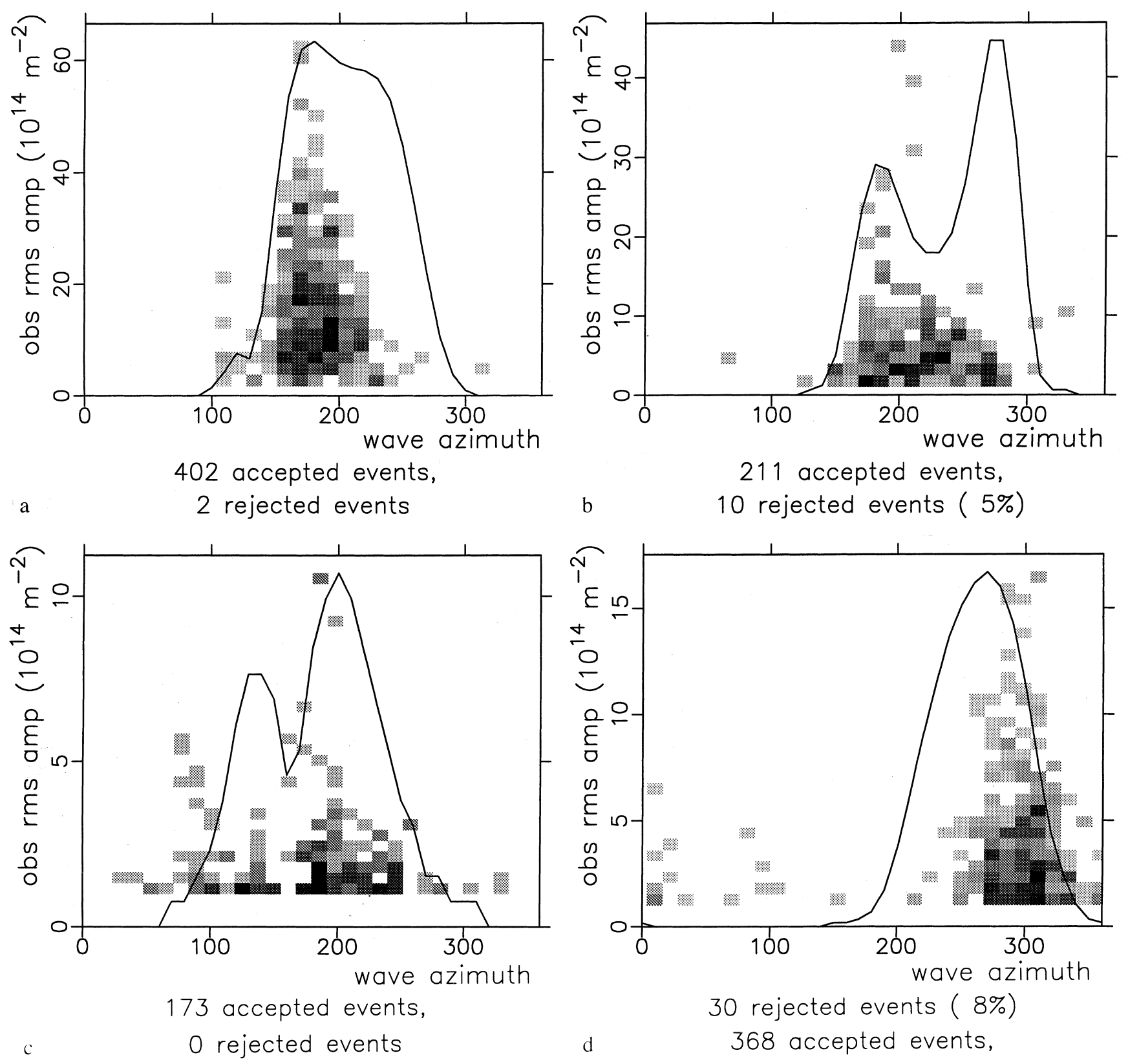

Fig. 4. As for Fig. 3 for GOES-2 in 1993-1994 (high satellite elevation).

values, with the modulus of the perturbation in electron density as weighting function, and are representative of the range of altitude contributing to the perturbation in TEC. Figure 2 gives grey-scale histograms of scatter plots of both coherence factor $f_{\text {coher }}$ and observed amplitudes versus calculated $a_{\mathrm{fs}}$ for all observed events with $0.4<f<1.2 \mathrm{mHz}, 100<\lambda<400 \mathrm{~km}$ and $V_{\phi}<$ $200 \mathrm{~ms}^{-1}$. For most events $a_{\mathrm{fs}}$ is in the range $\left(-20^{\circ},+20^{\circ}\right)$, and the largest intensities are for $a_{\mathrm{fs}}$ close to zero, which corresponds to $f_{\text {coher }}$ between $50 \%$ and $100 \%$. Thus the most intense observed events correspond to predicted favourable observing conditions.

\subsection{Azimuthal distribution of $A G W s$}

As explained in Sect. 4 we consider here data sets corresponding to (1) observing conditions (season, local time, line-of-sight orientation) as homogeneous as possible, and (2) to wave parameters typical of medium-scale AGWs. We simulate propagation of waves with the same azimuth, wavelength and frequency as in the observed data. Those which are revealed to become evanescent at some altitude are rejected from the statistics. For non-evanescent waves, grey-scale histograms of scatter plots of their observed amplitudes vs. propagation azimuths are produced, on to which are superimposed the predicted lobes (giving the sensitivity 

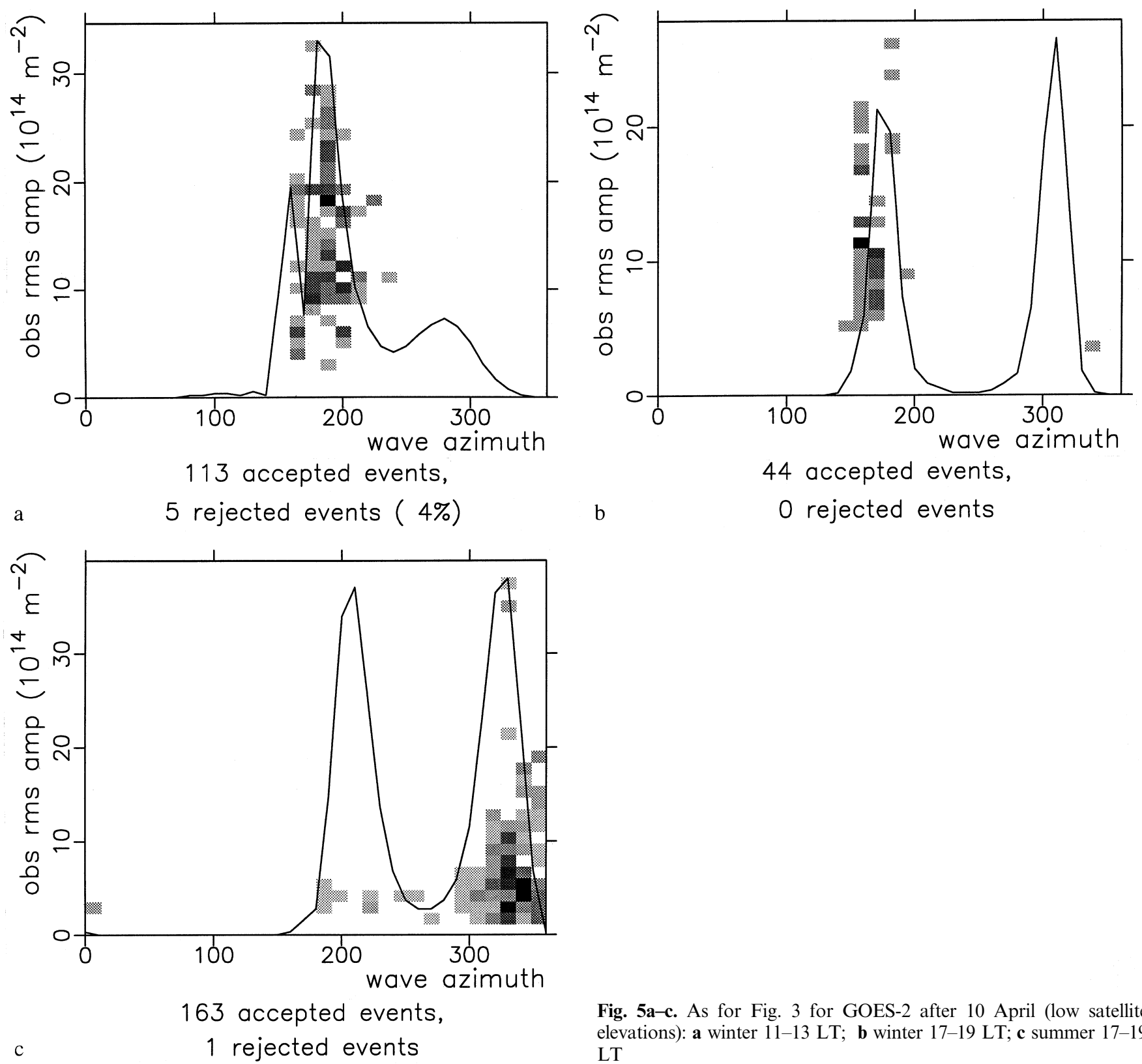

Fig. 5a-c. As for Fig. 3 for GOES-2 after 10 April (low satellite elevations): a winter 11-13 LT; b winter 17-19 LT; c summer 17-19 LT

of the observation as a function of the wave azimuth) calculated for (1) the mean line of sight of the selected data set and (2) average wave parameters $(f=0.7 \mathrm{mHz}$, $\lambda=200 \mathrm{~km})$.

Figures. 3-7 give such grey-scale histograms of the observed events in the 11-13 and 17-19 LT intervals in winter and summer for the four satellites. For each data selection the numbers of accepted (non-evanescent) and rejected (evanescent) events are indicated. The percentage of rejected events is always small $(0-8 \%)$. There are no events outside the lobes except for ATS-3 and GOES-2 93-94 in summer between 17 and 19 LT (Figs. 3d and $4 \mathrm{~d}$ ). In both cases these events are extremely weak $\left(<410^{14} \mathrm{~m}^{-2}\right)$.

These figures provide examples of both truncation by or incomplete filling of the lobe:

- In winter at noon, ATS-3 and GOES-2_93-94 show very similar distributions of strong events (Figs. 3a and 4a), the right (north) limits of which seem to be real, since the lobes extend more westwards than the observed distributions (incomplete filling). The left (east) limit is possibly also real, since there is hardly truncation for GOES-2_93-94 (Fig. 4a) whereas the lobe of ATS-3 seems to be incompletely filled on its left side (Fig. 3a).

- The distributions observed with GOES-2_95-96 (Fig. 5) are obviously truncated by the narrow lobes (complete filling of one or either lobe).

- The distributions observed in summer at 18 LT with GOES-2 93-94 (Fig. 4d) and GOES-3 (Fig. 7c) are truncated on their right sides near $350^{\circ}$ (east of north), but the lobes are incompletely filled on their left sides. 

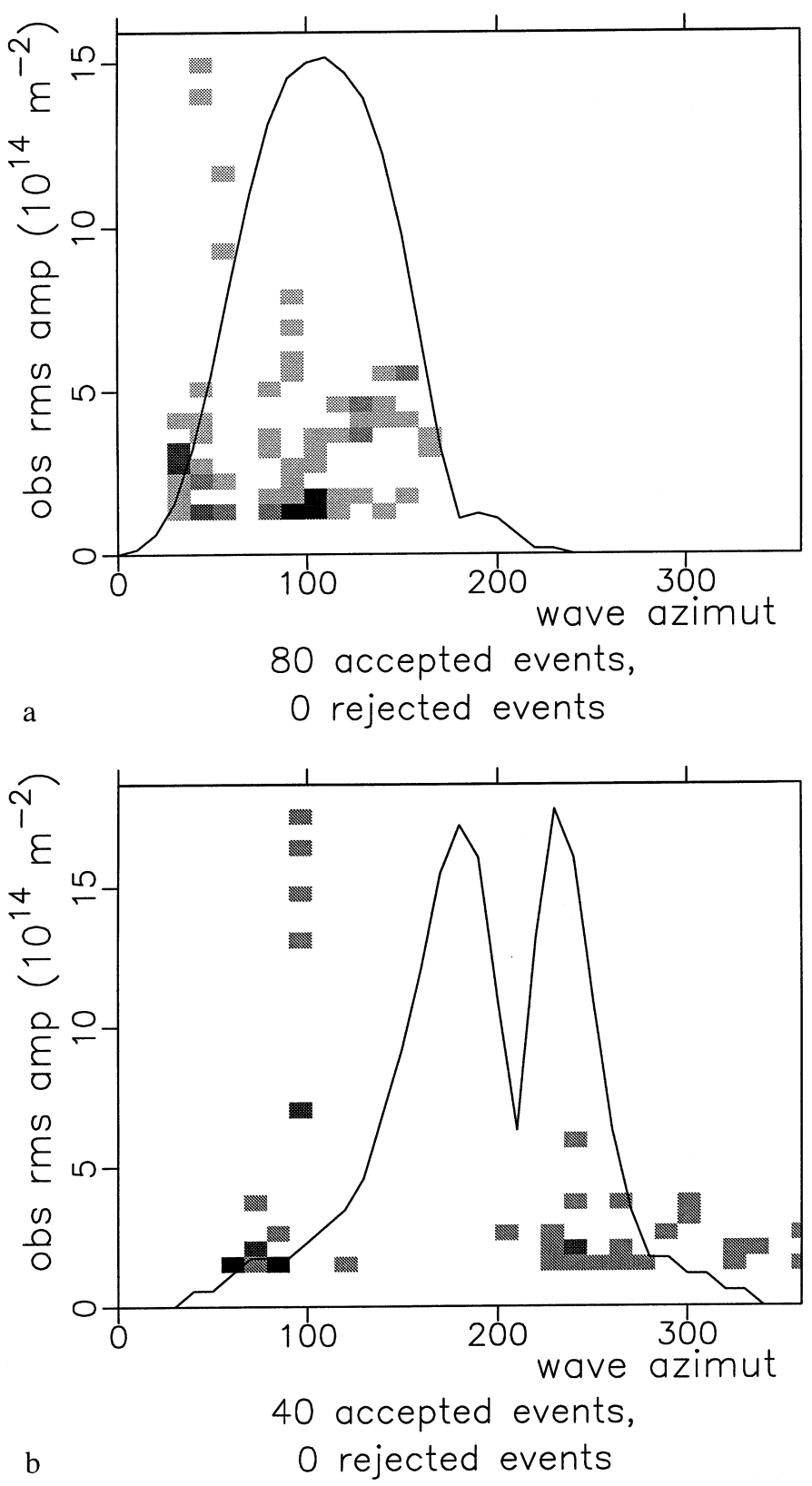

Fig. 6a, b. As for Fig. 3 for ATS-1 in summer: a 11-13 LT, b 17-19 LT

- The distributions for ATS-3 and GOES-2 93-94 in summer at noon (Figs. 3c and $4 \mathrm{c}$ ) are wider than in winter at the same time and practically fill the lobes; most of these events are weak, a result already known from previous studies (Jetal 95; Mercier, 1996).

The truncation effect of the observed azimuth distribution by the lobe corresponding to the line of sight is dramatic for GOES-2_95-96 (Fig. 5). The predicted lobe has two narrow peaks separated by a deep minimum, and the comparison with observations constitutes a strong constraint for the simulation model.

Distributions observed with ATS-3, GOES-2 and GOES-3 in winter at noon (Figs. 3a, 4a, 5a and 7a) correspond to the population $P_{1}$ of Jetal 95, with propagation azimuths around $180^{\circ}$. They are also observed, although with smaller amplitudes, in winter at 18 LT (Figs. 3b, 4b, 5b and 7b).

Distributions seen by GOES-2_93-94, GOES-2_95-96 and GOES-3 in summer at $18 \mathrm{LT}^{-}$(Figs. $4 \mathrm{~d}, 5 \mathrm{~d}, 7 \mathrm{c}$ ) have propagation azimuths between $300^{\circ}$ and $360^{\circ}$. They correspond to the population $P_{2}$ that Jetal 95 had inferred from observations of GOES-2_93-94 only. More northern propagation azimuths (up to $360^{\circ}$ ) can now be observed with GOES-2_95-96, up to the limit of its lobe. Note that the other few events of large amplitude in Fig. 5c coincide nearly with the eastern peak of the lobe. ATS-3, the azimuth of which is more southward than those of GOES-2 and GOES-3 does not observe efficiently the population $P_{2}$ (Fig. $3 \mathrm{~d}$ ).

The lobe of ATS- 1 in summer between 11 and 13 LT (Fig. 6a) is more eastward than that of any other satellite. It is filled with waves with propagation azimuths between $30^{\circ}$ and $180^{\circ}$, with low and moderate amplitudes. It is possible that this population is merely the extension of the background of the population $P_{1}$, which is less concentrated and less intense in summer than in winter (compare Figs. 3a and c, and 4a and c).

It must be noted that the daily variations in the pointing azimuths (see Table 1) are much smaller than the width of the observed azimuthal distributions of AGWs and do not allow us to investigate the effect of this parameter using only one satellite.

\subsection{Observed amplitude of $A G W$ s and elevation of the line of sight}

The pointing elevation is a sensitive parameter in the simulation of TEC perturbation (see Figs. A2, A3 and A4 in Mercier, 1996), and may strongly affect the integration along the line of sight. The daily variations in the elevations of ATS-3 (and to a lesser extent of GOES-2_93-94, see Table 1) are a large fraction of the range of possible elevations and allow us to follow continuously the effect of this parameter with one satellite, which is not the case for the pointing azimuth.

In particular one expects a zero or very small TEC perturbation for a line of sight parallel to the magnetic field $\boldsymbol{B}$ : the only means of varying TEC is then through recombination, since ionisation can move only along $\boldsymbol{B}$. This is nearly the case for observations with ATS-3 (which is near the meridian plane) at elevation close to the dip angle of $\boldsymbol{B}\left(\sim 60^{\circ}\right.$ near Los Alamos); there is however a magnetic declination of $12^{\circ}$ east. Figure 8 gives scatter plots of observed amplitudes vs. pointing elevation for ATS-3 and GOES-2_93-94 in winter between 11 and $13 \mathrm{LT}$. The superimposed curves give the predicted TEC perturbation at $12 \mathrm{LT}$ as a function of the elevation of the line of sight, for a wave with $f=0.7 \mathrm{mHz}, \lambda=200 \mathrm{~km}$ and $\alpha_{\text {wave }}=170^{\circ}$, typical of wave azimuths in Figs. $3 \mathrm{a}$ and $4 \mathrm{a}$. Although the ranges of line-of-sight elevation are not very large, these curves roughly mimic the observed distributions.

In order to improve the elevation range for a more marked effect, we must relax the selection on the local 

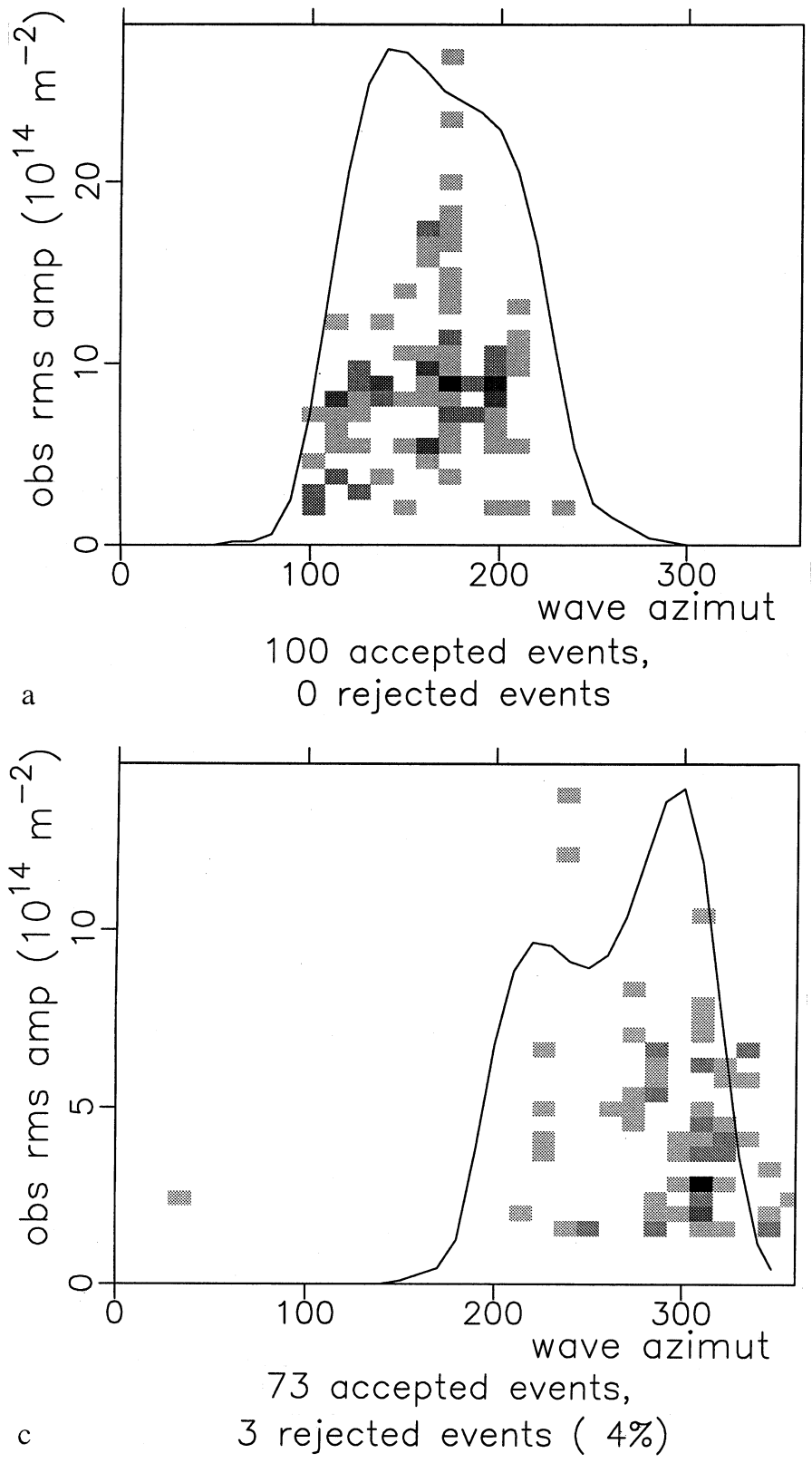

time, taking the risk of introducing diurnal variations. Diurnal effects can be reduced as much as possible in selecting waves with $\alpha_{\text {wave }}$ in the range $130^{\circ}-220^{\circ}$, belonging thus only to population $P_{1}$. Figure 9 gives the results for ATS-3 and GOES-2. The ranges of elevations are much larger and the predicted trends are similar to what is actually observed.

\subsection{Evanescent waves}

In all the selected sets of data, a small fraction $(\sim 4 \%)$ of waves was found to be evanescent when launched at an altitude $z_{\text {launch }}=20 \mathrm{~km}$, and they were rejected. As discussed in Sect. 2 this choice of $z_{\text {launch }}$ is partly for convenience, $20 \mathrm{~km}$ being the lower limit of validity of the adopted model for the unperturbed atmosphere. The

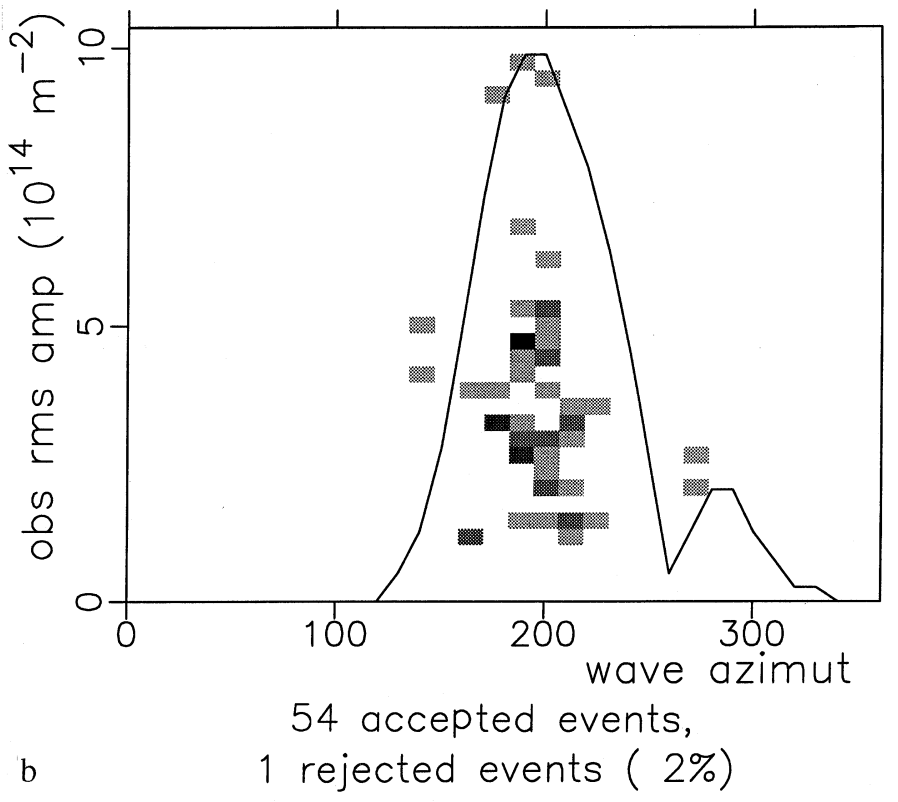

Fig. 7a-c. As for Fig. 3 for GOES-3: a winter 11-13 LT; b winter 17-19 LT; c summer 17-19 LT

fact that most of the selected events are not evanescent merely shows that they could propagate through the whole atmosphere. A complete study of the evanescent events is beyond the scope of this paper, since we focus here on typical medium-scale AGWs. We want only to point out some statistical properties of evanescent events. Figure 10 compares scatter plots of the observed amplitudes vs. wave azimuths for all non-evanescent and evanescent selected events: the fraction of rejected (evanescent events) is larger for azimuths near $290^{\circ}$, that is among events of the population $P_{2}$, which are mainly observed during evening in summer. Figure 11 shows that most of evanescent events have higher frequencies than non-evanescent ones, near the upper limit of our accepted frequency range, and that they have also somewhat larger trace speeds that nonevanescent ones. 

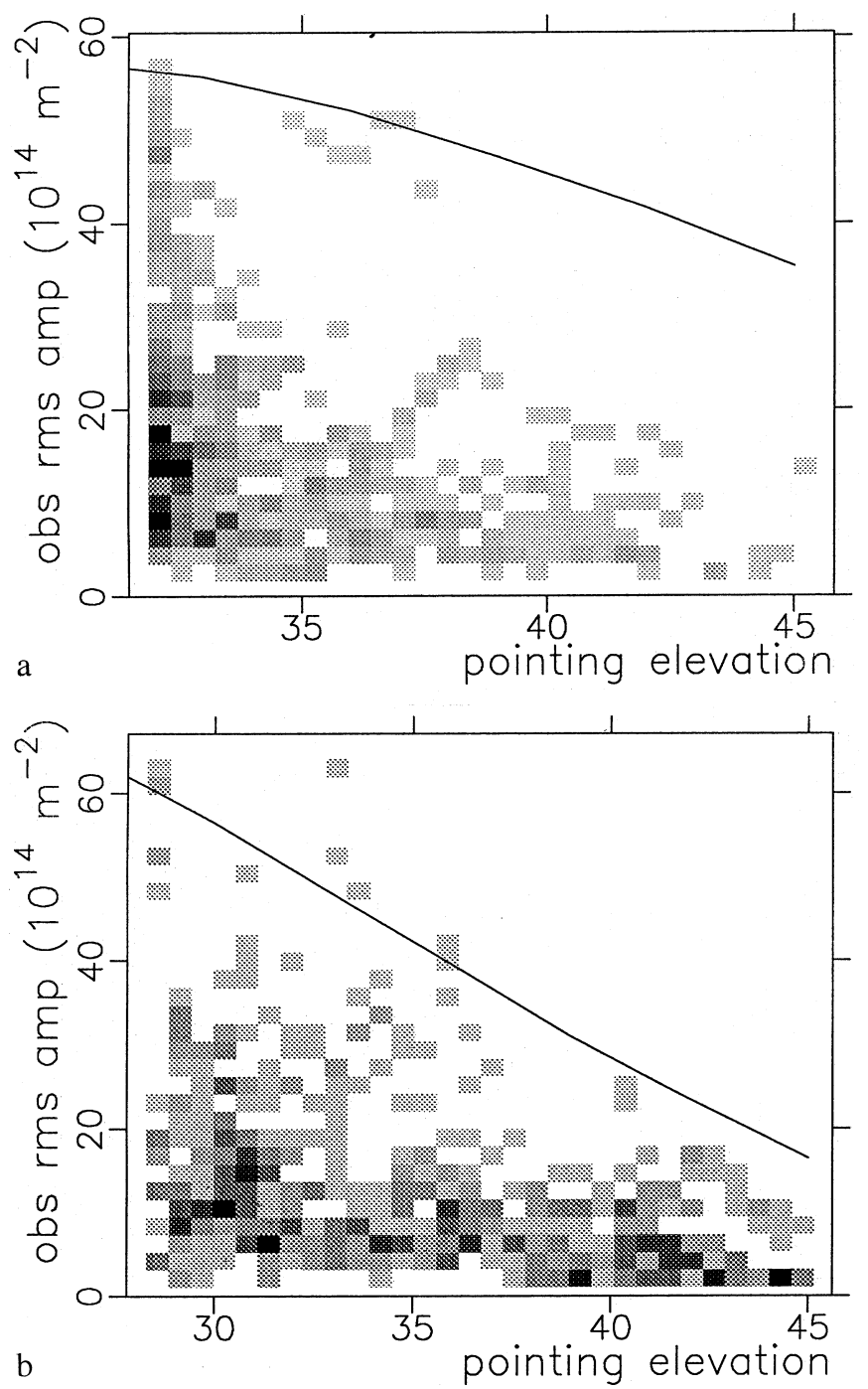

Fig. 8a,b. Grey-scale histograms of the elevation distribution of accepted (non-evanescent) events $(0.4 \mathrm{mHz}<f<1.2 \mathrm{mHz})$ observed in winter between 11 and 13 LT: a with ATS-3 (629 accepted events, 16 rejected events); b GOES-2_93-94 (501 accepted events, 25 ejected events). Superimposed curves are predicted responses to a wave with $f=0.7 \mathrm{mHz}, \lambda=200 \mathrm{~km}$ and $\alpha_{\text {wave }}=170^{\circ}$ at $12 \mathrm{LT}$

\section{Discussion and conclusion}

Results of the simulation show that observational results may be deeply biased by the observational technique. One must be very cautious when interpreting observational results, and the use of a simulation code is a necessary tool.

In our case, the use of only one satellite (with a weakly varying line of sight) eliminates drastically waves with propagation azimuths in wide angular ranges, essentially in the half plane opposite the satellite direction. This may result in a sharp cut off in the observed angular distribution of waves, since the criterion of detection is their amplitude.

In the case of methods combining techniques involving line-of-sight integration (interferometry, differential
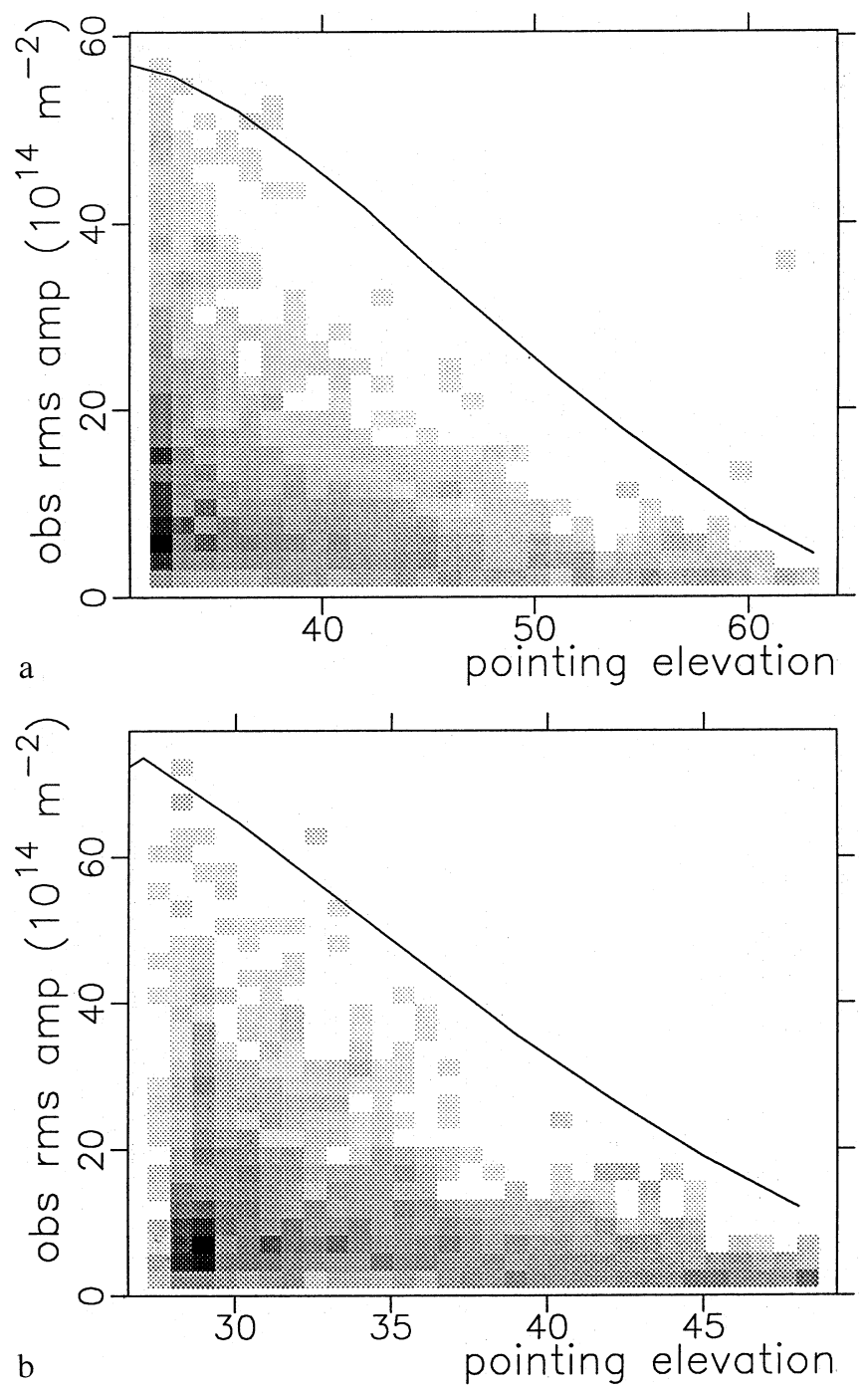

Fig. 9. Same as Fig. 8, events between 8 and 18 LT (3075 and 2518 accepted events, respectively, for ATS-3 and GOES-2)

Doppler shift and Faraday rotation), biases difficult to quantify may arise, namely in the propagation azimuths of TIDs if the lines of sight involved in the derivation of both components of the TEC gradient are not identical. This was already pointed out by van Velthoven et al. (1990).

The comparison of our observations and predictions supports the interpretation that most of the events we observed are due to AGWs:

- events are restricted to the predicted azimuth lobes in the distributions of the observed amplitudes according to wave azimuths and satellite elevations, except for a few faint events (Figs. 3d, 4d and 6b);

- the most intense events correspond to favourable observing conditions according to the simulation (Fig. 2), i.e. the line of sight makes a small angle $\left(<20^{\circ}\right)$ with the wave front;

- the fraction of evanescent events is small: $5 \%$ for all events and even less $(\leq 2 \%)$ during wintertime at noon. Most of these evanescent events have propagation azimuths in the NW quadrant and have high frequen- 

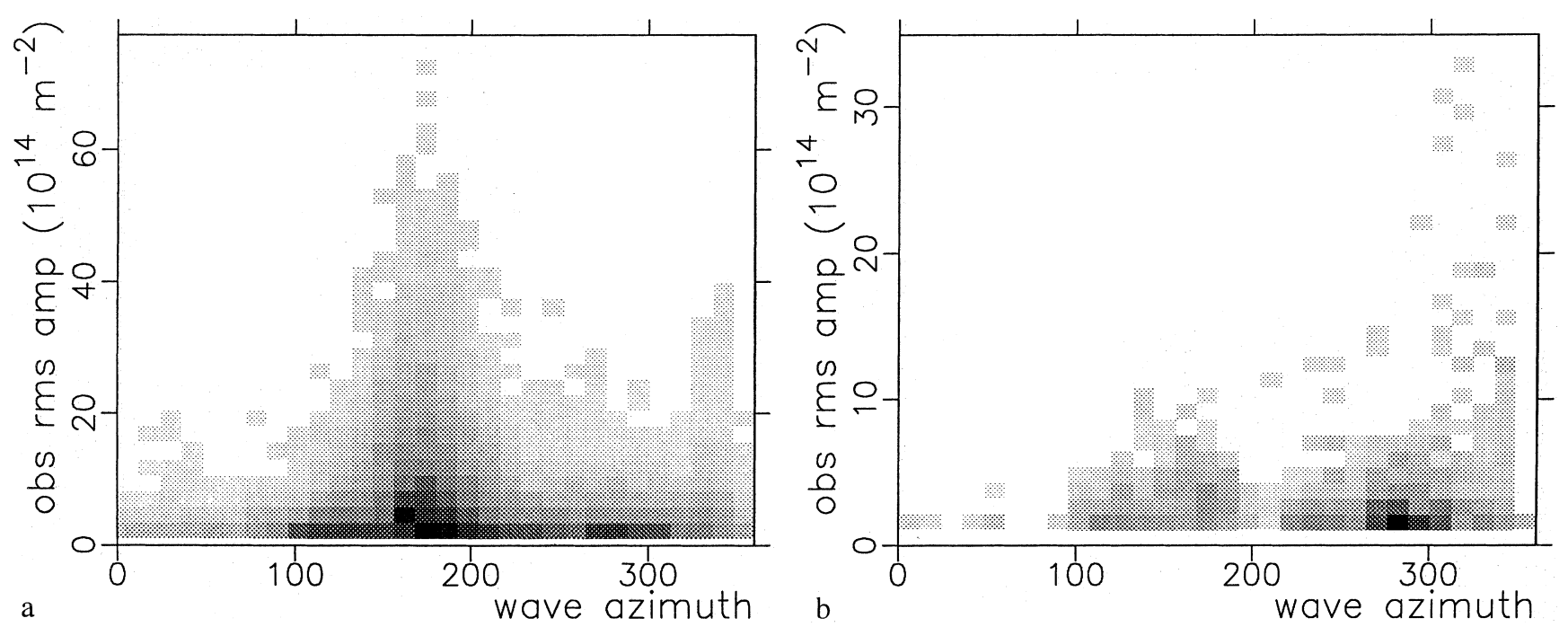

Fig. 10a, b. Grey-scale histograms of scatter plots of the observed amplitudes vs. wave azimuths for the same selected events as in Fig. 2:a 20672 non-evanescent events; b 1137 evanescent events

cies, close to the Brunt-Väisälä frequency (Fig. 11). The absence of events with low frequency and large trace speeds (more typical of large-scale AGWs) is due to our selection criteria, and particularly to the condition $V_{\phi}<200 \mathrm{~ms}$. The "evanescent" events in the NW quadrant may in fact be related to plasmaspheric irregularities (Jacobson et al., 1995b) rather than to AGWs. These events' feeble amplitudes and enhanced trace speeds are consistent with their being the lowfrequency extreme of this plasmaspheric phenomenon. The "propagation" azimuths of these events are also consistent with their being of plasmaspheric origin.

This consistency between observations and predictions allows one to use the simulation to discuss observational results, and particularly the azimuthal distribution of AGWs. We confirm the existence of two clearly separated populations $P_{1}$ and $P_{2}$ of medium-scale
AGWs, already inferred by Jetal 95 . The population $P_{1}$, essentially present during daytime in winter, is well observed with GOES-2 and ATS-3 (Figs. 3a and 4a). It exhibits a marked peak in the amplitude of observed waves between $140^{\circ}$ and $200^{\circ}(\mathrm{E}$ of $\mathrm{N})$. In winter this peak is narrower than the azimuthal lobes allowed by instrumental bias and is apparently not truncated for ATS-3 (Fig. 3a), and is only slightly truncated on the east side for GOES-2 (Fig. 4a). The east limit of the tail of the distribution cannot be specified because observations with ATS-1 are too scarce in winter. In summer this peak is less marked, and is wider. The instrumentally allowed azimuthal lobes are completely filled. The observations with ATS-1 (Fig. 6a), which also completely fill the predicted lobe, show that the actual azimuthal distribution of $P_{1}$ extends much further northwards, at least down to $30^{\circ}$.
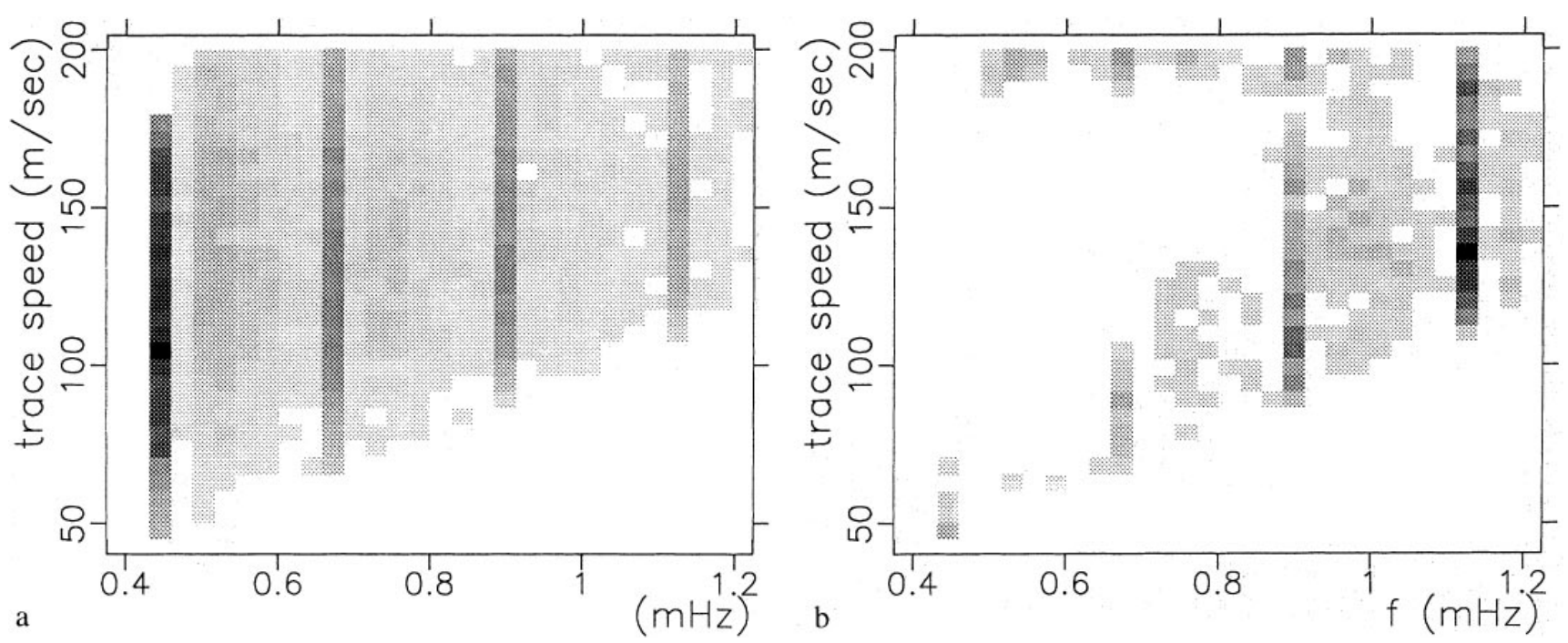

Fig. 11a, b. Grey-scale histograms of scatter plots of the trace speed vs. frequency for the same events as in Fig. 10: a 20672 non-evanescent events, b 1137 evanescent events. Darker vertical strips are artefacts resulting from preferential window durations before calculating spectra 
The population $P_{2}$, essentially present during the evening in summer, is not seen with ATS-3. Observations with GOES-2 93-94 show that the observed azimuthal distribution incompletely fills the predicted lobe on the west side, with a clear truncation on the north side (Fig. 4d). Later observations at low elevations with GOES-2 95-96 indicate that $P_{2}$ extends further northwards (Fig. 5d), but the limit cannot be yet specified.

The gap between $P_{1}$ and $P_{2}$, near $270^{\circ}$, was already known from Jetal 95 . We show here that, with the presently used satellites, the limits towards east and north of $P_{1}$ and towards north and east of $P_{2}$ are apparent and are due to the lack of sensitivity of the observational method to waves with these azimuths.

The main limitation in this study is the poor coverage in lines of sight, due to the small number of satellites used. Waves in the north-east quadrant can only be detected through observations with ATS-1, which are few. In addition there are "hidden relations" between some observation parameters (e.g. between elevation of the line of sight and local time and date) so it can be difficult to separate their influence. Thus climatology effects can be confused with geometry effects. It would thus be desirable to extend this study with more satellites, or at least with a larger data base for ATS-1 and GOES-3.

Acknowledgements. The authors wish to thank referees for their careful reading of the manuscript and their constructive comments. This work was essentially done when one of us (C. M.) was invited in the NIS1 group in Los Alamos; he is grateful to W. Vigil for his computer assistance.

Topical Editor D. Alcaydé thanks T.A.T. Spoelstra and F. Bertin for their help in evaluating this paper.

\section{References}

Alcaydé, D., An analytical static model of temperature and composition from 20 to $2000 \mathrm{~km}$ altitude, Ann. Géophys., 37, 4, 515-528, 1981

Bertel, L., F. Bertin, and J. Testud, De la mesure du contenu électronique intégré appliqué à l'observation des ondes de gravité de moyenne échelle, J. Atmos. Terr. Phys., 38, 261-270, 1976.
Bertin, F., J. Testud, L. Kersley, and P. R. Rees, The meteorological jet stream as a source of medium scale gravity waves in the thermosphere: an experimental study, J. Atmos. Terr. Phys., 40, 1161-1183, 1978.

Dalgarno, A., and F. J. Smith, The thermal conductivity and viscosity of atomic oxygen, Planet. Space Sci., 9, 281-282, 1962.

Hedin, A. E., N. W. Spencer, and T. L. Killeen, Empirical thermal model of upper thermosphere winds based on atmosphere and dynamics Explorer satellite data, J. Geophys. Res., 93 A9, 9959 9978, 1988.

Jacobson, A. R., and W. C. Erickson, Wavenumber resolved observations of ionospheric waves using the VLA, Planet. Space Sci., 40, 447-455, 1992.

Jacobson, A. R., R. S. Massey, and W. C. Erickson, A study of transionospheric refraction of radio waves using the Clark Lake radio observatory, Ann. Geophysicae 9, 546-552, 1991.

Jacobson, A. R., R. C. Carlos, R. S. Massey, and W. Guanghui, Observations of travelling ionospheric disturbances with a satellite-beacon radio-interferometer: Seasonal and local-time behavior, J. Geophys. Res., 100, 1653-1665, 1995a.

Jacobson, A. R., R. C. Carlos, R. S. Massey, W. Guanghui, and G. Hoogeveen, Geophys. Res. Lett., 22, 2461-2464, 1995b.

Jacobson, A. R., G. Hoogeveen, R. C. Carlos, G. Wu, B. G. Fejer, and M. C. Kelley, Observations of inner plasmaspheric irregularities with a satellite-beacon radio-interferometer array, J. Geophys. Res., 101, 19 655-19 682, 1996.

Mercier, C., Observation des Ondes de Gravité par RadioInterférométrie, in Actes du Séminaire EISCAT-ARCAD, Ed. P. Kaufman, 67-74, 1983.

Mercier, C., Observation of Atmospheric Gravity Waves by Radio-Interferometry, J. Atmos. Terr. Phys., 48, 605-624, 1986.

Mercier, C., Some characteristics of atmospheric gravity waves observed by radio-interferometry, Ann. Geophysicae 14, 42-58, 1996.

Mercier, C., F. Génova, and M. G. Aubier, Radio observations of atmospheric gravity waves, Ann Geophysicae, 7, 195-202, 1989.

Spoelstra, T. A. T., Combining TID observations: NNSS and radio interferometry data, J. Atoms. Terr. Phys., 54, 1185-1195, 1992.

Spoelstra, T. A. T., and H. Kelder, Effects produced by the ionosphere on radio-interferometry, Radio Sci, 19, 779-788, 1984.

Van Velthoven, P. F. J., C. Mercier and H. Kelder, Simultaneous observations of travelling ionospheric disturbances by twodimensional radio-interferometry and the differential Doppler technique applied to satellite signals, J. Atmos. Terr. Phys., 52, 305-312, 1990.

Volland, H., The upper atmosphere as a multiply refractive medium for neutral air motions, J. Atmos. Terr. Phys., 31, 491-514, 1969a.

Volland, H., Full wave calculation of gravity wave propagation through the thermosphere, J. Geophys. Res., 74, 1786-1795, $1969 b$. 